\title{
The shape of broad-line profiles in active galactic nuclei
}

\author{
W. Kollatschny and M. Zetzl \\ Institut für Astrophysik, Universität Göttingen, Friedrich-Hund Platz 1, 37077 Göttingen, Germany \\ e-mail: wkollat@astro.physik.uni-goettingen.de
}

Received 13 April 2012 / Accepted 5 November 2012

\section{ABSTRACT}

\begin{abstract}
Aims. We present a study of the broad optical/UV emission line profiles in active galactic nuclei (AGN) to get information on the dominant velocity components (turbulence, rotation, etc.) in the central broad-line region (BLR).

Methods. We introduce line broadening simulations of emission line profiles and compare these results with the largest homogeneous data set of reverberation-mapped AGN.

Results. The underlying broad-line profiles in AGN are Lorentzian profiles caused by turbulence in the line emitting region. The turbulent velocities are different for the different line emitting regions of $\mathrm{H} \gamma, \mathrm{H} \alpha$, Ly $\alpha, \mathrm{C}$ III] $\lambda 1909$, He II $\lambda 1640$, and $\mathrm{Si}$ IV $\lambda 1400$. The turbulent velocities go from $400 \mathrm{~km} \mathrm{~s}^{-1}$ for $\mathrm{H} \beta$ up to $3800 \mathrm{~km} \mathrm{~s}^{-1}$ for Ly $\alpha+\mathrm{N} \mathrm{v} \lambda 1240$. The dominating broadening mechanism of these profiles is broadening due to rotation. The rotation velocities causing the line profile broadening go from $500 \mathrm{~km} \mathrm{~s}^{-1}$ up to $6500 \mathrm{~km} \mathrm{~s}^{-1}$. Here we present interrelations between observed emission line widths (FWHM) and their related rotational velocities to correct for the contribution of the turbulence to the broad-line profiles.
\end{abstract}

Key words. accretion, accretion disks - line: profiles - galaxies: Seyfert - galaxies: active - quasars: emission lines

\section{Introduction}

It is now generally accepted that a super-massive black hole in the center of active galactic nuclei (AGN) is surrounded by an accretion disk (e.g. Urry \& Padovani 1995). In the outer regions of the accretion disk, the broad emission lines are created by photoionization. However, many details of this line emitting region are unknown, and there are many models that treat their geometry and structure (e.g. Collin-Souffrin et al. 1988; Emmering et al. 1992; Königl \& Kartje 1994; Murray \& Chiang 1997; Elvis 2000; Kollatschny 2003a; Ho 2008; Gaskell 2010; Goad et al. 2012, and references therein). This broad-line region (BLR) is spatially not resolved on direct images.

Information on the structure and kinematics of this region can in principle be obtained from the broad emission line profiles. However, there is the ambiguity in the possibility that different geometries and different kinematics result in emission line profiles that have similar shapes. This problem makes it difficult to disentangle the profile contribution of individual parameters (e.g. Netzer 1990; Sulentic et al. 2000; Zamfir et al. 2010, and references therein). The shape and the width of the emission line profiles in AGN might depend, among others parameters, on the velocity field, on the geometry of the line emitting gas, on obscuration effects, on the superposition of line emission from different regions, and on the isotropy/anisotropy of the line emission.

The velocity field might be a superposition of different components, such as Doppler motions, turbulence, shock components, in/outflow components, and rotation. Different velocity components result in different profiles, and the final profile is a convolution of different components. Doppler motions in the gravitational potential of a central black hole can result in Gaussian profiles, where the width of the lines reflects the Doppler motion of the line emitting gas (Peterson \& Wandel 1999; Fromerth \& Melia 2000; Peterson \& Wandel 2000). Logarithmic profiles agree well with observations of emission line wings and can be produced by outflowing gas (e.g. Blumenthal \& Mathews 1975; Netzer 1990). The existence of Lorentzian profiles is consistent with emission from extended accretion disks (Veron-Cetty et al. 2001; Sulentic et al. 2002). Goad et al. (2012) demonstrate that turbulent motions in the outer accretion disk produce Lorentzian profiles. Furthermore, Lorentzian profiles might be explained by shock breakouts in dense winds (Moriya \& Tominaga 2012). In addition to these base-line profiles, both rotation and electron scattering (e.g. $\mathrm{H} \alpha$ in NGC 4593; Laor 2006) can lead to broadening of the line profiles.

Osterbrock pointed out as early as 1978 that a combination of turbulence with rotation agrees well with the distribution of observed line widths of Seyfert 1 galaxies known at that time. More recently, researchers have explored the possibility of an additional net infall component (Gaskell 2010, for a review; Hu et al. 2012; but see Sulentic et al. 2012) and an additional outflow component in luminous AGNs (e.g. Wang et al. 2011).

The contribution of the velocity components might be different from line to line, as well as from galaxy to galaxy. In their study of AGN line profiles Robinson et al. (1995) demonstrated that different types of models produce similar kinds of profiles and that the profile shapes differ between the AGN populations. In addition, the optical and UV emission line profiles are similarly diverse in appearance. In an investigation of broad emission lines from the Sloan Digital Sky Survey (SDSS) Richards et al. (2002) point out that the explanation of their profiles is one of the most important tasks of future AGN models. Using CIV emission line profiles, Richards et al. (2011) show that the differences in the accretion disk wind between quasars can account for some of the diversity of broad emission line profiles.

We demonstrated in a first paper (Kollatschny \& Zetzl 2011, hereafter called Paper I) that there is a general trend between the full-width at half maximum (FWHM) and the line width ratio FWHM $/ \sigma_{\text {line }}$ in the broad emission lines of AGN. Different 
Table 1. H $\gamma, \mathrm{H} \alpha$, Ly $\alpha, \mathrm{C}$ III] $\lambda 1909$, Si IV $\lambda 1400$, and He II $\lambda 1640$ line widths of our AGN sample.

\begin{tabular}{|c|c|c|c|c|}
\hline Object & Line & $\begin{array}{c}F W H M \\
{\left[\mathrm{~km} \mathrm{~s}^{-1}\right]}\end{array}$ & $\begin{array}{c}\sigma \\
{\left[\mathrm{km} \mathrm{s}^{-1}\right]}\end{array}$ & $F W H M / \sigma$ \\
\hline $\begin{array}{l}\text { PG1229+204 } \\
\end{array}$ & $\mathrm{H} \gamma$ & $3207 \pm 875$ & $1540 \pm 159$ & $2.082 \pm 0.169$ \\
\hline PG0844+349 & $\mathrm{H} \gamma$ & $4946 \pm 1085$ & $2058 \pm 218$ & $2.403 \pm 0.195$ \\
\hline PG0052+251 & $\mathrm{H} \gamma$ & $5633 \pm 3585$ & $2230 \pm 502$ & $2.526 \pm 0.432$ \\
\hline PG0953+414 & $\mathrm{H} \gamma$ & $2960 \pm 589$ & $1299 \pm 193$ & $2.279 \pm 0.261$ \\
\hline PG1307+085 & $\mathrm{H} \gamma$ & $4278 \pm 881$ & $1758 \pm 193$ & $2.433 \pm 0.204$ \\
\hline PG2130+099 & $\mathrm{H} \gamma$ & $2661 \pm 481$ & $1836 \pm 191$ & $1.449 \pm 0.130$ \\
\hline PG1226+023 & $\mathrm{H} \gamma$ & $3274 \pm 484$ & $1688 \pm 142$ & $1.940 \pm 0.130$ \\
\hline PG0804+761: & $\mathrm{H} \gamma$ & $2201 \pm 295$ & $1256 \pm 141$ & $1.752 \pm 0.160$ \\
\hline PG1211+143: & $\mathrm{H} \gamma$ & $2014 \pm 249$ & $1376 \pm 157$ & $1.464 \pm 0.143$ \\
\hline PG0844+349 & $\mathrm{H} \alpha$ & $2436 \pm 329$ & $1625 \pm 73$ & $1.499 \pm 0.057$ \\
\hline PG1426+015 & $\mathrm{H} \alpha$ & $5450 \pm 842$ & $4254 \pm 290$ & $1.281 \pm 0.078$ \\
\hline PG1229+204 & $\mathrm{H} \alpha$ & $3229 \pm 364$ & $1737 \pm 118$ & $1.859 \pm 0.101$ \\
\hline PG1617+175 & $\mathrm{H} \alpha$ & $3794 \pm 780$ & $2483 \pm 160$ & $1.528 \pm 0.083$ \\
\hline PG1411+442 & $\mathrm{H} \alpha$ & $1877 \pm 375$ & $2437 \pm 196$ & $0.770 \pm 0.072$ \\
\hline PG0026+129 & $\mathrm{H} \alpha$ & $1117 \pm 109$ & $1961 \pm 135$ & $0.570 \pm 0.056$ \\
\hline PG0804+761 & $\mathrm{H} \alpha$ & $3155 \pm 569$ & $2046 \pm 138$ & $1.542 \pm 0.088$ \\
\hline PG2130+099 & $\mathrm{H} \alpha$ & $1574 \pm 438$ & $1421 \pm 80$ & $1.108 \pm 0.059$ \\
\hline NGC 4151 & $\mathrm{H} \alpha$ & $3156 \pm 300$ & $2422 \pm 79$ & $1.303 \pm 0.038$ \\
\hline NGC 4593 & $\mathrm{H} \alpha$ & $3399 \pm 196$ & $1253 \pm 90$ & $2.713 \pm 0.147$ \\
\hline NGC 7469 & $\mathrm{H} \alpha$ & $1615 \pm 119$ & $1164 \pm 68$ & $1.387 \pm 0.071$ \\
\hline NGC 3516 & $\mathrm{H} \alpha$ & $4770 \pm 893$ & $2108 \pm 69$ & $2.263 \pm 0.057$ \\
\hline NGC 3227 & $\mathrm{H} \alpha$ & $3168 \pm 67$ & $1977 \pm 134$ & $1.602 \pm 0.091$ \\
\hline PG0052+251: & $\mathrm{H} \alpha$ & $2682 \pm 453$ & $1913 \pm 85$ & $1.402 \pm 0.054$ \\
\hline NGC 4151: & $\mathrm{H} \alpha$ & $3724 \pm 529$ & $1721 \pm 47$ & $2.164 \pm 0.046$ \\
\hline PG1211+143: & $\mathrm{H} \alpha$ & $1425 \pm 382$ & $2321 \pm 231$ & $0.614 \pm 0.083$ \\
\hline PG1226+023: & $\mathrm{H} \alpha$ & $1638 \pm 424$ & $2075 \pm 239$ & $0.789 \pm 0.104$ \\
\hline PG1307+085: & $\mathrm{H} \alpha$ & $3084 \pm 1041$ & $1843 \pm 98$ & $1.673 \pm 0.073$ \\
\hline Mrk 279: & $\mathrm{H} \alpha$ & $3408 \pm 555$ & $1405 \pm 266$ & $2.426 \pm 0.351$ \\
\hline NGC 5548: & $\mathrm{H} \alpha$ & $3044 \pm 381$ & $1694 \pm 80$ & $1.797 \pm 0.069$ \\
\hline Fairall9 & $\operatorname{Ly} \alpha$ & $3503 \pm 1474$ & $4120 \pm 308$ & $0.850 \pm 0.069$ \\
\hline 3C 390.3 & $\operatorname{Ly} \alpha$ & $8732 \pm 985$ & $3952 \pm 203$ & $2.210 \pm 0.088$ \\
\hline 3C 390.3: & $\operatorname{Ly} \alpha$ & $8225 \pm 781$ & $4600 \pm 141$ & $1.788 \pm 0.044$ \\
\hline NGC 5548 & $\mathrm{C}_{\text {IIII }} \lambda 1909$ & $4895 \pm 1263$ & $3227 \pm 176$ & $1.517 \pm 0.070$ \\
\hline NGC 5548 & С III] $\lambda 1909$ & $5018 \pm 1458$ & $2360 \pm 222$ & $2.126 \pm 0.156$ \\
\hline NGC 7469 & Si IV $\lambda 1400$ & $6033 \pm 1112$ & $3495 \pm 269$ & $1.726 \pm 0.109$ \\
\hline NGC 3783 & Si IV $\lambda 1400$ & $6343 \pm 2021$ & $3488 \pm 161$ & $1.819 \pm 0.068$ \\
\hline NGC 5548 & Si IV $\lambda 1400$ & $7044 \pm 1849$ & $4014 \pm 253$ & $1.755 \pm 0.090$ \\
\hline NGC 5548 & Si IV $\lambda 1400$ & $6455 \pm 3030$ & $2576 \pm 389$ & $2.506 \pm 0.288$ \\
\hline NGC 7469 & He II $\lambda 1640$ & $10725 \pm 1697$ & $3723 \pm 113$ & $2.881 \pm 0.065$ \\
\hline NGC 3783 & Не II $\lambda 1640$ & $8008 \pm 1268$ & $3870 \pm 162$ & $2.069 \pm 0.068$ \\
\hline NGC 5548 & Не II $\lambda 1640$ & $8929 \pm 1571$ & $4397 \pm 154$ & $2.031 \pm 0.056$ \\
\hline NGC 5548 & He II $\lambda 1640$ & $9803 \pm 1594$ & $3897 \pm 264$ & $2.516 \pm 0.130$ \\
\hline
\end{tabular}

Notes. Measurements of objects with colons (:) were considered as less reliable by the authors (Peterson et al. 2004).

emission lines exhibit different systematics in the FWHM $/ \sigma_{\text {line }}{ }^{-}$ vs.-FWHM diagram. The line width FWHM reflects the rotational motion of the broad-line gas in combination with the associated turbulent motion. This turbulent velocity is different for the different emission lines.

Here we present a further study of additional broad optical and UV emission line profiles of reverberation-mapped AGN. The line profiles are parameterized by the ratio of their FWHM to their line dispersion $\sigma_{\text {line }}$.

\section{Data: the line profile sample}

We studied in detail the mean profiles, as well as the root-meansquare (rms) profiles of the $\mathrm{H} \beta$, He II $\lambda 4686$, and C IV $\lambda 1550$ lines of an AGN sample in Paper I. This set of observations is the largest homogeneous sample of variable AGN at this time, and it is based on reverberation-mapped AGN spectra (Peterson et al. 2004). The optical spectra were collected with different ground based telescopes while the UV spectra were taken with the IUE satellite and the Hubble Space Telescope. The sample has the advantage that all the spectra were reduced in exactly the same way and that all spectra of each galaxy were intercalibrated with respect to each other. The original AGN sample consists of 37 objects. In some cases multiple line spectra exist for a particular galaxy based on different variability campaigns of the same galaxy.

The present study is based on this data set as well. Here we analyze all the remaining optical and UV broad-line profiles we did not consider in Paper I, i.e., the $\mathrm{H} \gamma, \mathrm{H} \alpha$, Ly $\alpha$, He II $\lambda 1640$, C III] $\lambda 1909$, and S IV $\lambda 1400$ lines.

Usually the observed broad-line profiles in AGN are more or less contaminated by additional narrow emission line components from the narrow line region. For avoiding any major ambiguity we only inspected the root-mean-square (rms) line profiles out of the sample of Peterson (2004) as we did in Paper I. The rms profiles display the clean profiles of the variable broad emission lines. The narrow line components disappear in these spectra because they are constant on time scales of years. The optical and UV emission line profiles of our AGN sample are parameterized by their line widths (FWHM, and $\left.\sigma_{\text {line }}\right)$. The relationship between FWHM and $\sigma_{\text {line }}$ contains information on the shape of the profile. We use all those line profiles of the sample that were regarded as both reliable and less so by Peterson (2004) - same as in Paper I.

All spectral data we are using in this current investigation are listed in Table 1 . We present $\mathrm{H} \gamma, \mathrm{H} \alpha$, Ly $\alpha, \mathrm{C}$ III] $\lambda 1909$, 
Si IV $\lambda 1400$, and He II $\lambda 1640$ line widths (FWHM) and line width ratios $(\mathrm{FWHM} / \sigma)$ of all galaxies in the AGN sample.

\section{Results}

The following are the emission line profiles resulting from various kinematical and dynamical models that have been discussed in the context of the BLR in AGNs:

- Gaussian profiles due to Doppler motions:

$$
F W H M / \sigma_{\text {line }}=2.35 \text {; }
$$

- Lorentzian profiles due to turbulent motions:

$$
F W H M / \sigma_{\text {line }} \approx 1(\rightarrow 0)
$$

- Exponential profiles caused by electron scattering:

$$
F W H M / \sigma_{\text {line }}=\sqrt{2} \ln 2 \approx 0.98
$$

- Logarithmic profiles caused by in-/outflow motions:

$$
F W H M / \sigma_{\text {line }} \approx 1(\rightarrow 0) \text {. }
$$

We present in Fig. 1 all these emission line profiles scaled to the same $\mathrm{H} \beta$ line width (FWHM). The exact mathematical value of FWHM $/ \sigma_{\text {line }}$ goes to zero for Lorentzian profiles because of the infinitely extended wings (see Fig. 1). However, the typical FWHM/ $/ \sigma_{\text {line }}$ values we are measuring are on the order of one when integrating the spectral lines over a few hundred Angstroms. Similarly the exact FWHM $/ \sigma_{\text {line }}$ value for logarithmic profiles cannot be calculated because the central intensity goes to infinity. See also discussions of this ratio in Peterson et al. (2004), Collin et al. (2006), and Goad et al. (2012).

In Fig. 2 we again show the basic logarithmic $\mathrm{H} \beta$ profile for $v_{\min } / v_{\max }=0.003$, as well as more line profiles generated by expanding spherical shells of radiatively-driven isotropically emitting clouds with different $v_{\min } / v_{\max }$ values ranging from 0.01 up to 0.9999 (see also Capriotti et al. 1980).

\subsection{Line profile broadening simulations}

We are trying to find appropriate answers to the following questions with our present investigation of AGN emission line profiles: which line profile is emitted intrinsically and what broadening mechanisms (besides the instrumental broadening) have an impact on the observed profile. Lorentzian emission line profiles and/or Gaussian profiles are the most accepted profiles that are thought to be emitted intrinsically.

We showed in Paper I that rotational line broadening is indeed the most important broadening parameter for AGN emission line profiles. We calculated the rotational line broadening by the convolution of Lorentzian or Gaussian profiles with ellipsoidal profiles. The rotational velocity $b=\Delta \lambda / x$ is by definition the half width at zero intensity (HWZI) of an ellipsoidal profile

$A(x)=\frac{2}{\pi} \sqrt{1-x^{2}}$.

In Paper I we used a numerical code that was developed by Hubeny et al. (1994) to compute the line broadening due to rotation. This program considers limb darkening as well. In the present study we use our own routine without limb darkening that is based on the following broadening formula:

$S(y)=\int_{-\infty}^{+\infty} W(y-x) A(x) \mathrm{d} x$
(Unsoeld 1955), where $W$ is the intrinsic line profile without rotational broadening, $A$ the rotational profile, and $S$ the convolved profile.

Other intrinsic line profiles and other broadening mechanisms than rotation lead to different profile shapes. We computed these profile shapes as well and compared them with observed AGN profiles.

Figures 3 and 4 show computed Lorentzian profiles that were broadened by rotational motions. The rotation velocities range from $100 \mathrm{~km} \mathrm{~s}^{-1}$ to $8000 \mathrm{~km} \mathrm{~s}^{-1}$. For the intrinsic $\mathrm{H} \beta$ Lorentzian profile we adopted a turbulent velocity of $500 \mathrm{~km} \mathrm{~s}^{-1}$ (see Paper I) in the line emitting region (Fig. 3). For the intrinsic Lorentzian profile of the C IV $\lambda 1549$ line we adopted a turbulent velocity of the line emitting region of $3000 \mathrm{~km} \mathrm{~s}^{-1}$ (Fig. 4). We present additional line broadened profiles in Figs. 5 and 6 . In Fig. 5 we made the assumption that the intrinsic $\mathrm{H} \beta$ profile is a Gaussian profile $\left(F W H M=500 \mathrm{~km} \mathrm{~s}^{-1}\right)$ that is broadened by rotation as in Fig. 3. Figure 6 demonstrates the line broadening of a Lorentzian $\mathrm{H} \beta$ profile owing to Doppler motions (convolution with a Gaussian profile). In all cases we made the assumption that the integrated line intensities remain constant.

\subsection{Observed and modeled line width ratios $F W H M / \sigma_{\text {line }}$ versus line width FWHM}

In the next step we present a comparison of the observed line profile shapes in different AGN spectra with computed line profiles.

\subsubsection{Rotational line broadening of Lorentzian $\mathrm{H} \beta$, He II $\lambda$ 4686, C IV $\lambda 1549$ profiles}

In Paper I (their Figs. 1-3) we showed the observed $\mathrm{H} \beta$, He II $\lambda 4686$, and C IV $\lambda 1549$ line width ratios $\mathrm{FWHM} / \sigma_{\text {line }}$ versus their line width FWHM in AGN. These line width data were taken from the sample of Peterson et al. (2004). We likewise included those measurements that were regarded as less reliable by the authors because they follow exactly the same trend. We modeled the observations by rotationally broadened Lorentzian profiles. We present in Fig. 7 the observed ratios $\mathrm{FWHM} / \sigma_{\text {line }}$ vs. FWHM for the $\mathrm{H} \beta$, He II $\lambda 4686$, and C IV $\lambda 1549$ profiles in one single plot, as well as their modeling with our new routine. The rotational velocities in this figure are slightly shifted towards higher FWHM (in comparison to Paper I) because the effect of limb darkening leads to a slightly modified rotational profile (see Fig. 168 in Unsoeld 1955). The data for these three line profiles and their corresponding models fill different areas in this plot. The underlying $\mathrm{H} \beta$ Lorentzian profile has a line width of $400 \mathrm{~km} \mathrm{~s}^{-1}$, the He II $\lambda 4686$ has an intrinsic line width of $900 \mathrm{~km} \mathrm{~s}^{-1}$, and the C IV $\lambda 1549$ line has an intrinsic line width of $2900 \mathrm{~km} \mathrm{~s}^{-1}$. These line widths are least square fits to the data in Fig. 7.

The modeled rotational velocities of the $\mathrm{H} \beta$ lines range from 500 to $6000 \mathrm{~km} \mathrm{~s}^{-1}$ in the individual galaxies. The He II $\lambda 4686$ and C IV $\lambda 1549$ lines exhibit higher rotational velocities of at least $1500 \mathrm{~km} \mathrm{~s}^{-1}$.

\subsubsection{Fitting the observed $\mathrm{H} \beta$ line width ratios with other models}

We generated further test models (Figs. 8 to 10) to adjust the observed $\mathrm{H} \beta$ line width ratios by means of additional intrinsic line profiles and/or further line broadening mechanisms. Figure 8 


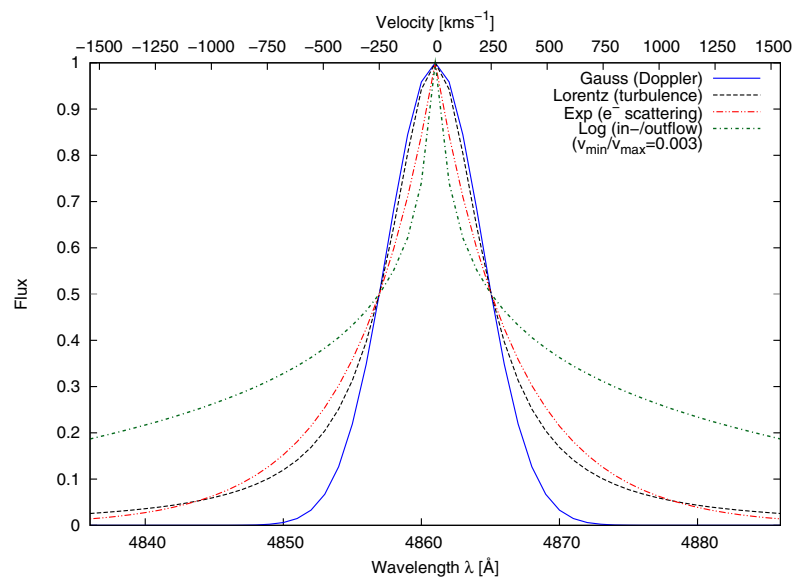

Fig. 1. Basic emission line profiles resulting from different kinematical models for the BLR in AGN: Gaussian (solid line, blue), Lorentzian (dashed, black), exponential (dot dot dashed, red), and logarithmic (dot dashed, green) profiles. All profiles are scaled to the same $\mathrm{H} \beta$ line width $\left(F W H M=500 \mathrm{~km} \mathrm{~s}^{-1}\right)$.

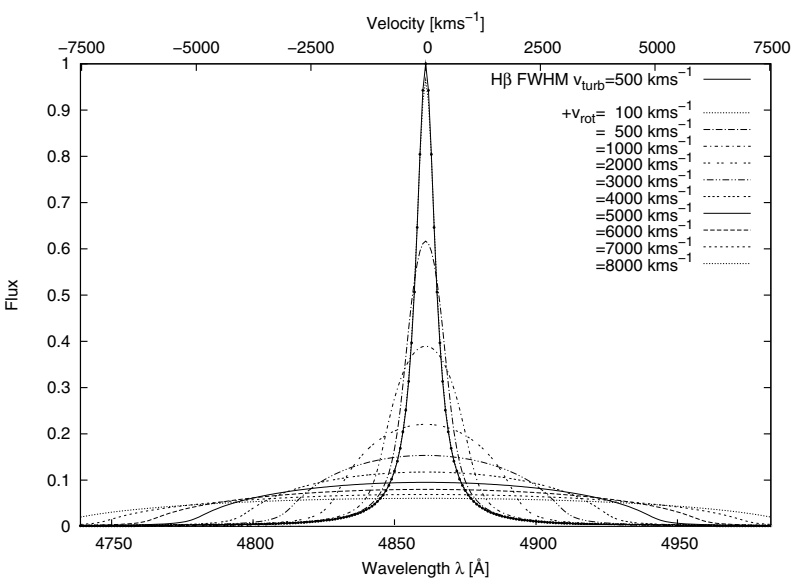

Fig. 3. Line broadening of a Lorentzian $\mathrm{H} \beta$ profile due to rotation. The rotation velocities range from $100 \mathrm{~km} \mathrm{~s}^{-1}$ to $8000 \mathrm{~km} \mathrm{~s}^{-1}$. The intrinsic turbulent velocity of the $\mathrm{H} \beta$ line corresponds to $500 \mathrm{~km} \mathrm{~s}^{-1}$. All profiles are normalized to have the same total flux.

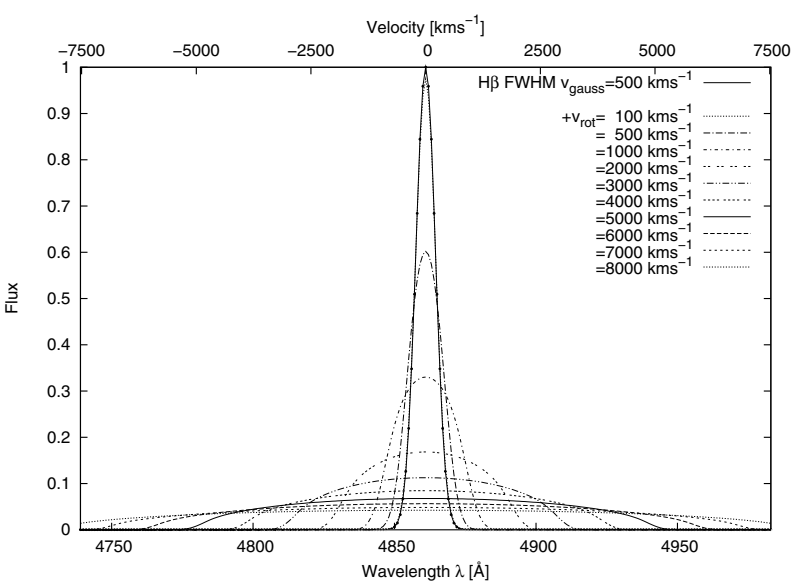

Fig. 5. Line broadening of a Gaussian $\mathrm{H} \beta$ profile due to rotation. The rotation velocities range from $100 \mathrm{~km} \mathrm{~s}^{-1}$ up to $8000 \mathrm{~km} \mathrm{~s}^{-1}$. The intrinsic line width of the $\mathrm{H} \beta$ line corresponds to $500 \mathrm{~km} \mathrm{~s}^{-1}$.

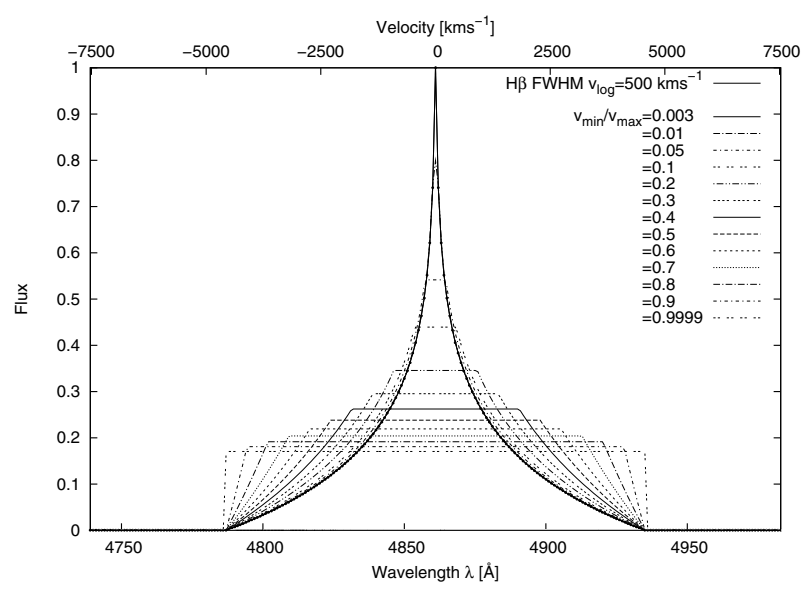

Fig. 2. Logarithmic $\mathrm{H} \beta$ profile with an intrinsic line width (FWHM) corresponding to $500 \mathrm{~km} \mathrm{~s}^{-1}$. Furthermore, we calculated the emission line profiles caused by expanding spherical shells of radiativelydriven isotropically emitting clouds for different values of $v_{\min }$ to $v_{\max }$ (see also Capriotti et al. 1980).

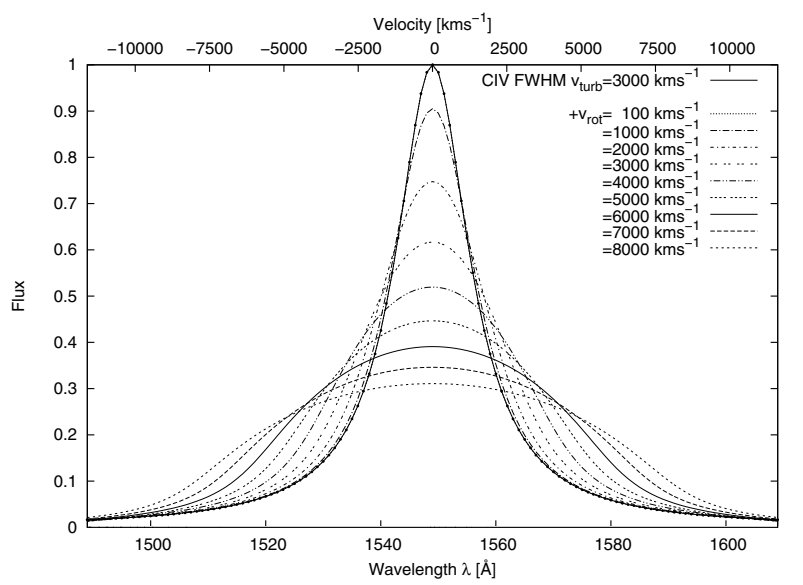

Fig. 4. Line broadening of a Lorentzian C IV $\lambda 1549$ profile due to rotation. The rotation velocities range from $100 \mathrm{~km} \mathrm{~s}^{-1}$ up to $8000 \mathrm{~km} \mathrm{~s}^{-1}$. The intrinsic turbulent velocity of the C IV $\lambda 1549$ line corresponds to $3000 \mathrm{~km} \mathrm{~s}^{-1}$.

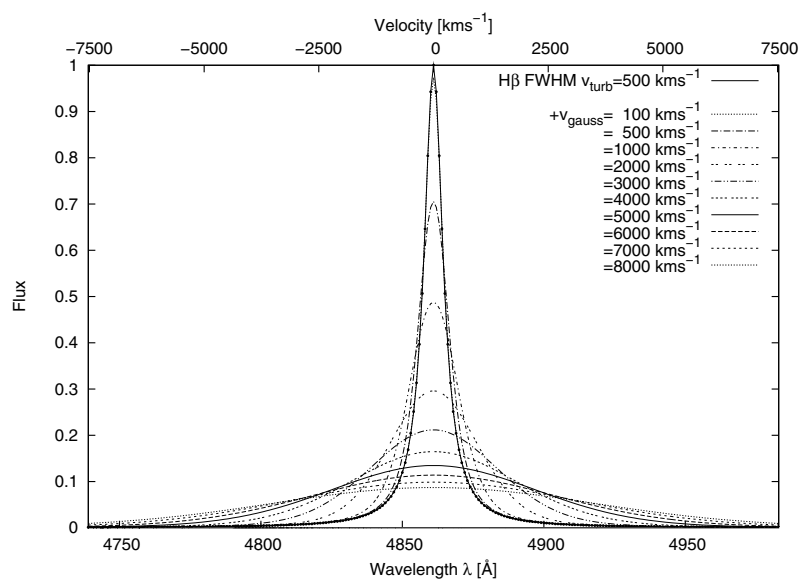

Fig. 6. Line broadening of a Lorentzian $\mathrm{H} \beta$ profile due to Doppler motions. The intrinsic line width of the $\mathrm{H} \beta$ line corresponds to $500 \mathrm{~km} \mathrm{~s}^{-1}$. This Lorentzian profile has been convolved with Gaussian profiles having widths of $100 \mathrm{~km} \mathrm{~s}^{-1}$ up to $8000 \mathrm{~km} \mathrm{~s}^{-1}$. 
W. Kollatschny and M. Zetzl: Shape of AGN line profiles

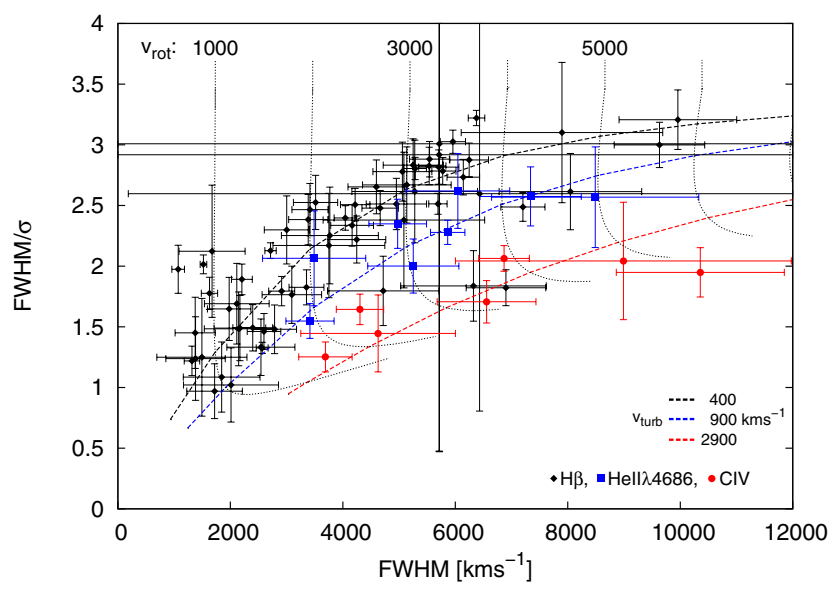

Fig. 7. Observed line width ratios FWHM/ $\sigma_{\text {line }}$ vs. line width FWHM for $\mathrm{H} \beta$ (black diamonds), He II $\lambda 4686$ (blue squares), and C IV $\lambda 1549$ (red circles). The dashed curves represent their corresponding theoretical line width ratios based on rotational line broadened Lorentzian profiles $\left(F W H M=400,900\right.$, and $\left.2900 \mathrm{~km} \mathrm{~s}^{-1}\right)$. The rotation velocities go from 500 to $7000 \mathrm{~km} \mathrm{~s}^{-1}$ (curved dotted lines from left to right). A color version of this figure is available in the online journal.

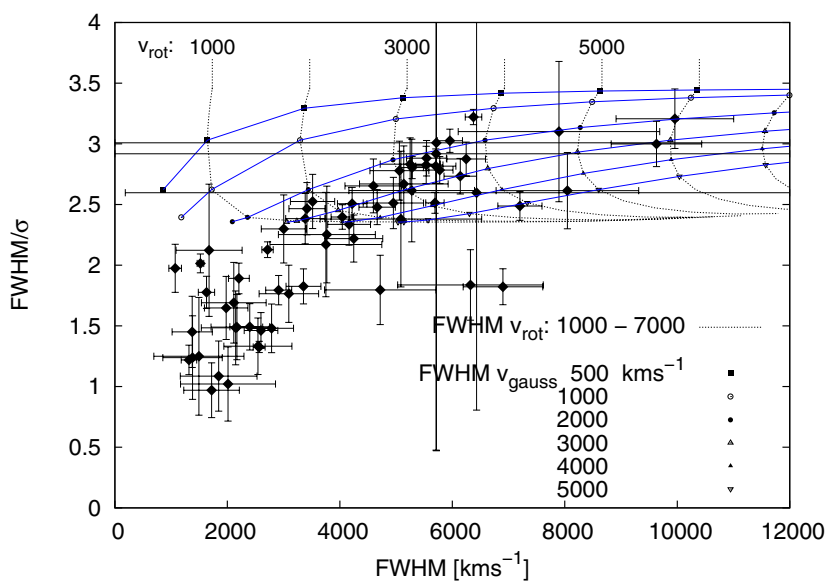

Fig. 8. Observed $H \beta$ line width ratios FWHM/ $\sigma_{\text {line }}$ vs. line width FWHM. The curves represent their modeling by rotational broadening $\left(500-7000 \mathrm{~km} \mathrm{~s}^{-1}\right)$ of Gaussian profiles $(500,1000,2000,3000,4000$, $5000 \mathrm{~km} \mathrm{~s}^{-1}$ line width). A color version of this figure is available in the online journal.

shows the computed trend of intrinsic Gaussian profiles that are broadened by rotation. The corresponding line profiles are presented in Fig. 5. The intrinsic Gaussian profiles had line widths (FWHM) ranging from 500 to $5000 \mathrm{~km} \mathrm{~s}^{-1}$ (from top to bottom). The rotational velocities go from 500 to $7000 \mathrm{~km} \mathrm{~s}^{-1}$. It is evident that these computed $\mathrm{H} \beta$ line width ratios cannot explain the observations because these broadened Gaussian profiles always exceed FWHM $/ \sigma_{\text {line }}$ ratio values of 2.35. Lorentzian profiles, on the other hand, can easily explain low $\mathrm{FWHM} / \sigma_{\text {line }}$ ratios as seen in Fig. 7.

Further line broadening tests of Lorentzian profiles convolved with Gaussian profiles that have widths of 500 to $10000 \mathrm{~km} \mathrm{~s}^{-1}$ explain neither the high observed FWHM $/ \sigma_{\text {line }}$ ratios nor the observed general trend of the $\mathrm{FWHM} / \sigma_{\text {line }}$ to line width FWHM ratios (Fig. 8). The corresponding line profiles are given in Fig. 6.

Figure 10 shows the trend of both observed and modeled $\mathrm{H} \beta$ line width ratios $\mathrm{FWHM} / \sigma_{\text {line }}$ versus $\mathrm{FWHM}$ for logarithmic profiles. We modeled logarithmic profiles for different $v_{\min } / v_{\max }$

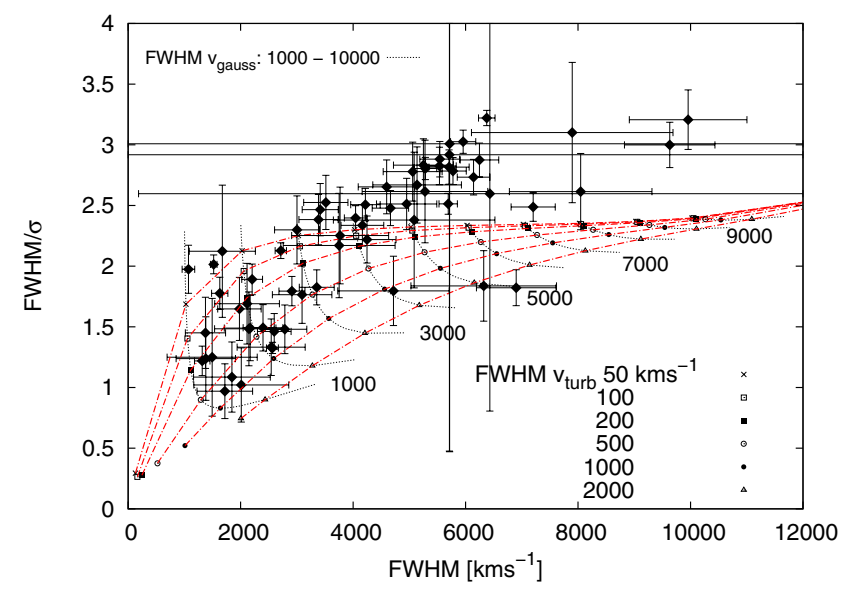

Fig. 9. Observed $H \beta$ line width ratios FWHM/ $\sigma_{\text {line }}$ vs. line width FWHM. The curves represent their modeling by convolving Lorentzian profiles $\left(50,100,200,500,1000,2000 \mathrm{~km} \mathrm{~s}^{-1}\right.$ line width) with Gaussian profiles (500-10000 $\mathrm{km} \mathrm{s}^{-1}$ line width). A color version of this figure is available in the online journal.

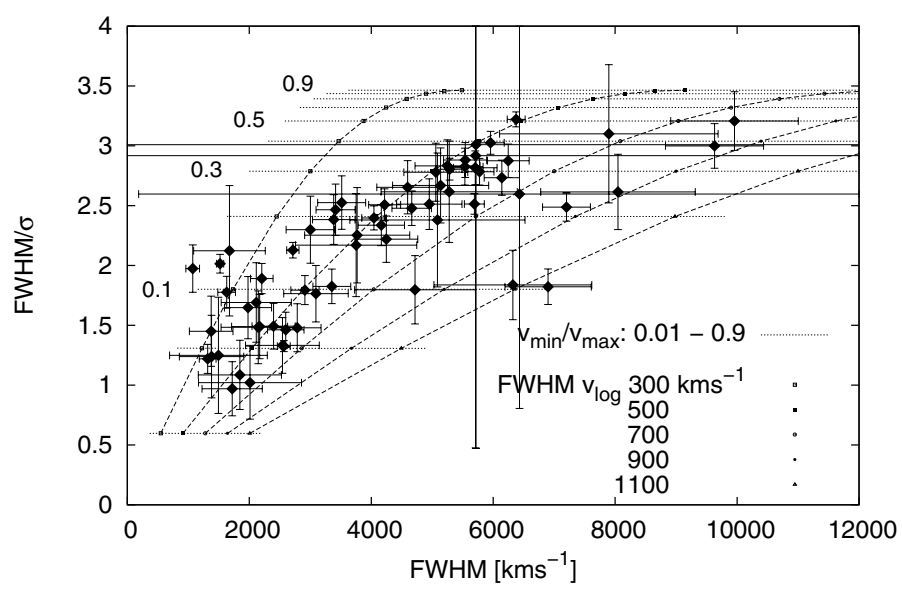

Fig. 10. Observed $H \beta$ line width ratios $\mathrm{FWHM} / \sigma_{\text {line }}$ vs. line width FWHM. The curves represent their modeling by logarithmic profiles for different $v_{\min } / v_{\max }$ ratios (0.01-0.9, from bottom to top). Furthermore, we varied the line width from $300,500,700,900$, to $1100 \mathrm{~km} \mathrm{~s}^{-1}$ (left to right).

ratios (0.01-0.9, from bottom to top). In addition we varied the line widths from $300,500,700,900$, to $1100 \mathrm{~km} \mathrm{~s}^{-1}$ (left to right). The corresponding line profiles are given in Fig. 2.

An exponential profile has a similar shape to a Lorentzian profile, especially in their line wings (see Fig. 1). Line broadening due to rotation of these two profile types leads to similar trends in the FWHM $/ \sigma_{\text {line }}$ vs. FWHM figures. Exponential profiles have a fixed $\mathrm{FWHM} / \sigma_{\text {line }}$ ratio of $\mathrm{FWHM} / \sigma_{\text {line }}=$ $\sqrt{2} \ln 2 \approx 0.9803$, while this ratio goes to zero for Lorentzian profiles. All in all, the observed trend of varying line shape as a function of line width - from the narrowest to the broadest line profiles - cannot be explained by one varying parameter alone.

\subsubsection{Rotational line broadening of Lorentzian $\mathrm{H} \gamma, \mathrm{H} \alpha$, Ly $\alpha$, C III] $\lambda$ 1909, He II $\lambda$ 1640, and Si IV $\lambda 1400$ profiles}

All observed rms emission line width ratios $\mathrm{FWHM} / \sigma_{\text {line }}$ versus line width FWHM (from Table 1), as well as their modeling, are shown separately in Figs. 11 to 16 for the different emission 


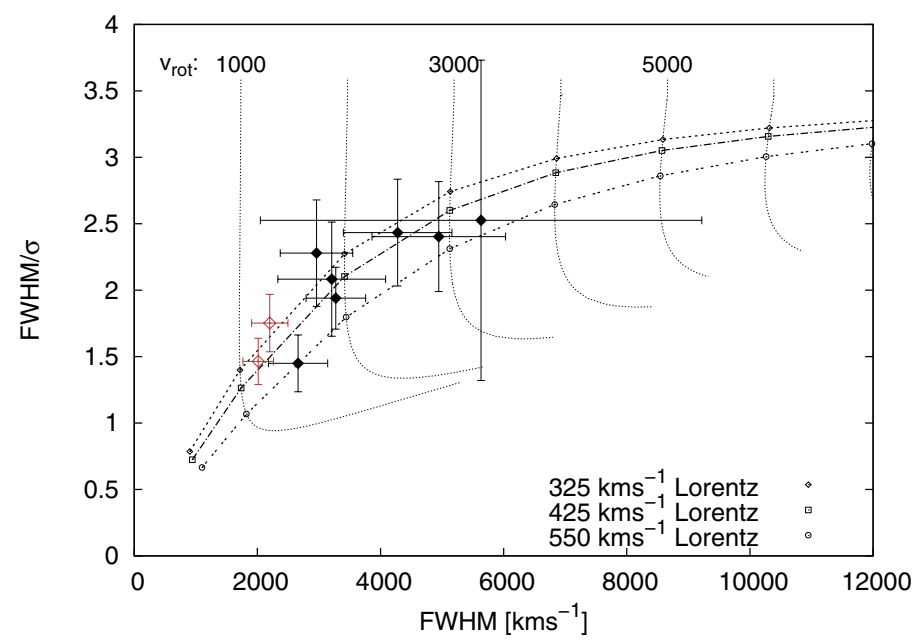

Fig. 11. Observed $H \gamma$ line width ratios $\mathrm{FWHM} / \sigma_{\text {line }}$ versus line width FWHM and their modeling by rotational broadening of Lorentzian profiles. Less reliable measurements are marked by open red diamonds.

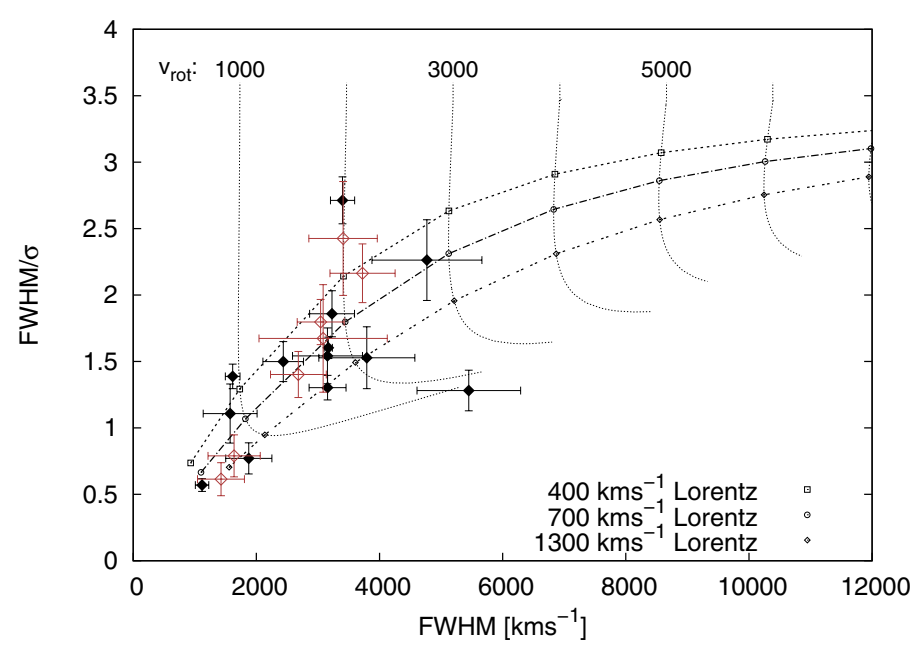

Fig. 12. Observed and modeled $H \alpha$ line width ratios $\mathrm{FWHM} / \sigma_{\text {line }}$ versus line width FWHM.

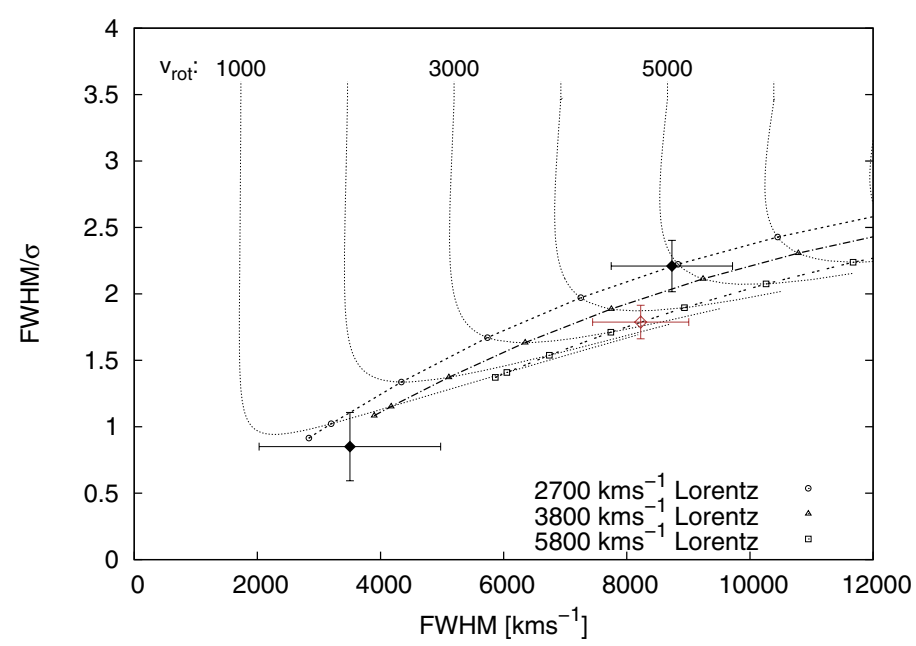

Fig. 13. Observed and modeled $L y \alpha+\mathrm{N} v \lambda 1240$ line width ratios FWHM/ $/ \sigma_{\text {line }}$ versus line width FWHM.

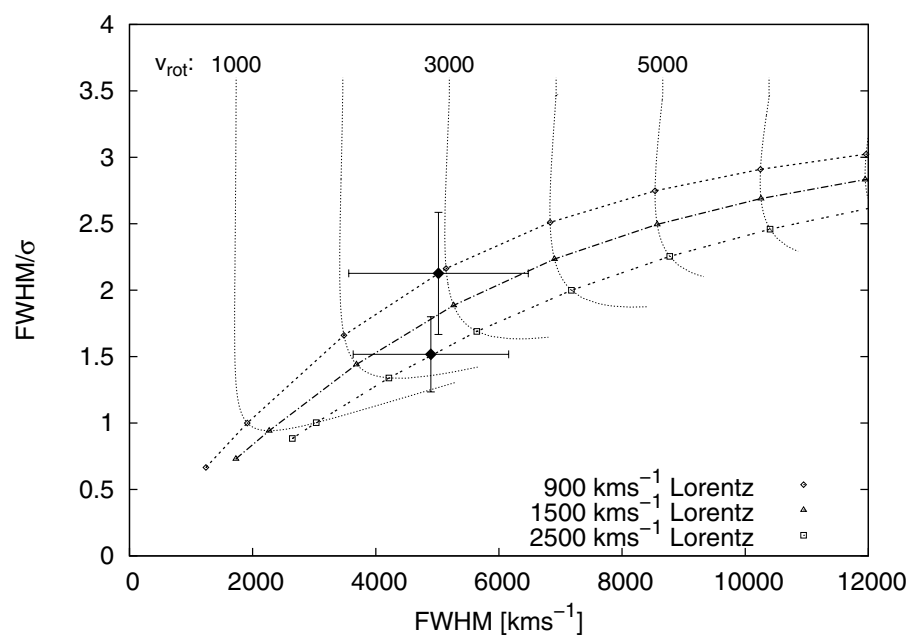

Fig. 14. Observed and modeled CIII] $\lambda 1909$ line width ratios $\mathrm{FWHM} / \sigma_{\text {line }}$ versus line width FWHM.

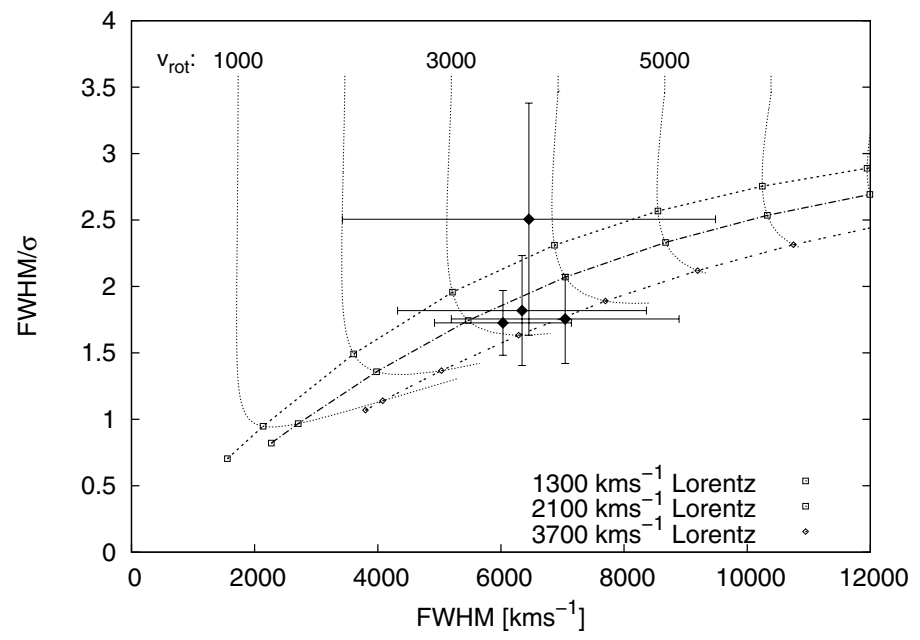

Fig. 15. Observed and modeled Si IV $\lambda 1400$ line width ratios $\mathrm{FWHM} / \sigma_{\text {line }}$ versus line width FWHM.

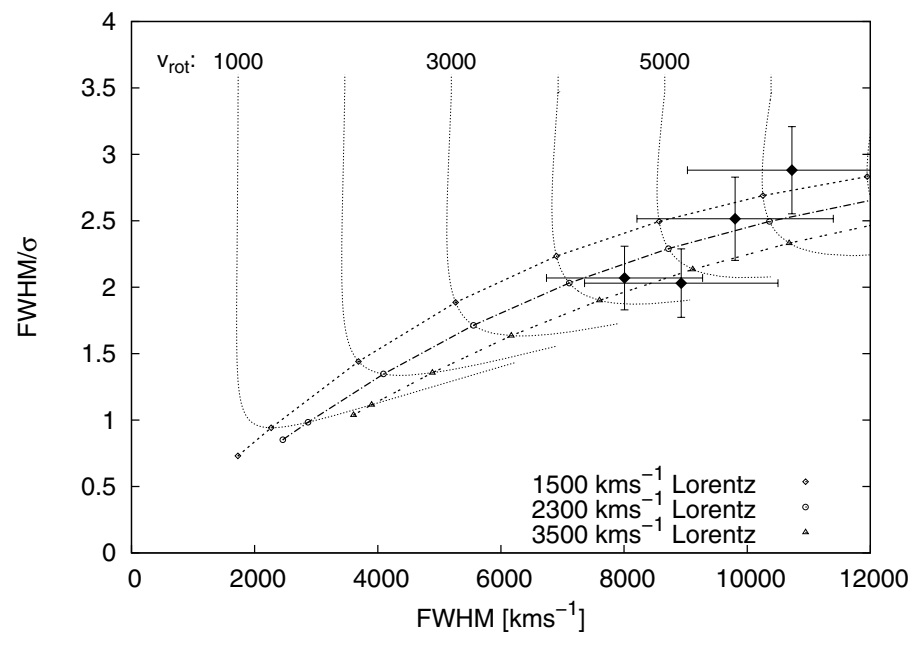

Fig. 16. Observed and modeled He II $\lambda 1640$ line width ratios $\mathrm{FWHM} / \sigma_{\text {line }}$ versus line width FWHM. 
lines $\mathrm{H} \gamma, \mathrm{H} \alpha$, Ly $\alpha, \mathrm{C}$ III] $\lambda 1909$, He II $\lambda 1640$, and Si IV $\lambda 1400$. The observed data are taken from the AGN sample (see Peterson et al. 2004). Those measurements that were regarded as less reliable by the authors are marked by open red diamonds. These less reliable measurements follow in most cases the general trend. Besides the least square fits we show lower and upper limits to the widths of the Lorentzian profiles (in Figs. 11 to 16). These limits are calculated from the variation in the individual data points with respect to the least square fit.

There are two $\mathrm{H} \alpha$ measurements not following the trend: the galaxies NGC 4593 and PG 1426+015. The galaxy NGC 4593 shows a high line width ratio $\mathrm{FWHM} / \sigma_{\text {line }}$ of 2.7 . This might be explained with a high-inclination angle of the accretion disk (see Paper I) in this galaxy. PG $1426+015$ shows a low $\mathrm{FWHM} / \sigma_{\text {line }}$ line width ratio with respect to the derived line width of $5450 \mathrm{~km} \mathrm{~s}^{-1}$. The same galaxy was an outlier in the corresponding $\mathrm{H} \beta$ figure (see Paper I).

Furthermore, there is one Si IV $\lambda 1400$ measurement (Fig. 15) that does not follow the general trend by showing a high $\mathrm{FWHM} / \sigma_{\text {line }}$ line width ratio. However, the error of this line measurement in the galaxy NGC 5548 is very large. A second measurement of this line in NGC 5548 at $\mathrm{FWHM} / \sigma_{\text {line }}=$ 1.755 (see Table 1) corresponds with the measurements of other galaxies.

The line width ratios FWHM/ $\sigma_{\text {line }}$ versus line width FWHM of all these optical and UV emission lines can be modeled in a simple way by rotational line broadening of Lorentzian profiles, as presented before in Paper I for the $\mathrm{H} \beta$, He II $\lambda 4686$, and C IV $\lambda 1550$ lines. To each emission line belongs a dedicated turbulent velocity that can be derived from the underlying Lorentzian profile. This specific turbulent velocity of the individual lines is the same in all galaxies. The intrinsic turbulent velocities belonging to the individual emission lines are listed in Table 2.

In Paper I we considered the possible effect of an inclination of the accretion disk. An inclination leads to smaller line widths in comparison to the intrinsic line widths. Here we did not correct for this in Table 2.

\section{Discussion}

\subsection{AGN line profiles}

In early studies of emission line profiles e.g. Blumenthal \& Matthews (1975) or Capriotti et al. (1980) fitted logarithmic profiles to observed quasar emission lines. However, later on it was noticed that their logarithmic fits result in line wings that are too small in comparison to the observed profiles. In those early years only very few high-quality AGN spectra existed.

Afterwards it became apparent that the profiles of narrowline Seyfert 1 galaxies with $H \beta$ line widths (FWHM) of less than $2000-4000 \mathrm{~km} \mathrm{~s}^{-1}$ are well adjusted by single Lorentzian profiles (Sulentic et al. 2000 and references therein; Veron et al. 2001; Marziani et al. 2003). These authors confirmed previous claims that observed broad Balmer line profiles are more precisely fitted by Lorentzian rather than Gaussian profiles. Gaussian profiles cannot reproduce the profiles observed in narrow-line Seyfert 1 galaxies.

Laor (2006) found evidence of exponential line wings in the $\mathrm{H} \alpha$ line of the low luminosity narrow-line Seyfert 1 galaxy NGC 4395. However, this galaxy is unique in having a broad $\mathrm{H} \alpha$ line width (FWHM) of $<520 \mathrm{~km} \mathrm{~s}^{-1}$ only. Laor (2006) explains this specific line profile by electron scattering in the broad-line region.
Table 2. Intrinsic turbulent velocities connected to the line emitting regions of the strongest emission lines.

\begin{tabular}{ll}
\hline \hline Emission line & $\begin{array}{l}\text { Turbulent velocity } \\
{\left[\mathrm{km} \mathrm{s}^{-1}\right]}\end{array}$ \\
\hline $\mathrm{H} \beta$ & $400(-175)(+300)$ \\
$\mathrm{H} \gamma$ & $425(-100)(+125)$ \\
$\mathrm{H} \alpha$ & $700(-300)(+600)$ \\
$\mathrm{He}$ II $\lambda 4686$ & $900(-300)(+200)$ \\
$\mathrm{C}$ III $\lambda 1909$ & $1500(-600)(+1,000)$ \\
$\mathrm{Si}$ IV $\lambda 1400$ & $2100(-800)(+1,600$ \\
$\mathrm{He}$ II $\lambda 1640$ & $2300(-800)(+1.200)$ \\
C IV $\lambda 1549$ & $2900(-1000)(+1400)$ \\
$\mathrm{Ly} \alpha+\mathrm{N}$ v $\lambda 1240$ & $3800(-1100)(+2000)$ \\
\hline
\end{tabular}

Observed $\mathrm{H} \beta$ profiles in AGN with line widths $(\mathrm{FWHM})<4000 \mathrm{~km} \mathrm{~s}^{-1}$ never can be explained by single Gaussian profiles or by a combination of Gaussian profiles. Gaussian profiles have a constant $\mathrm{FWHM} / \sigma_{\text {line }}$ ratio of 2.35 . In contrast, all observed $\mathrm{H} \beta$ profiles showing line widths (FWHM) $<4000 \mathrm{~km} \mathrm{~s}^{-1}$ exhibit lower FWHM/ $\sigma_{\text {line }}$ ratios (see Fig. 8). A combination of Lorentzian profiles with Gaussian profiles does not match the observed trend in the FWHM $/ \sigma_{\text {line }}$ versus line width FWHM plots (see Fig. 8). Logarithmic profiles meet the observed trend in the $\mathrm{FWHM} / \sigma_{\text {line }}$ versus $\mathrm{FWHM}$ plots for those line profiles with line widths $F W H M<6000 \mathrm{~km} \mathrm{~s}^{-1}$ (Fig. 10). However, broader profiles cannot be modeled in a simple way by varying only one parameter.

The profiles are not the same in all the quasars. Sulentic et al. (2000) call those quasars having $\mathrm{H} \beta$ line widths (FWHM) > $4000 \mathrm{~km} \mathrm{~s}^{-1}$ Population B quasars in contrast to Population A quasars having $\mathrm{H} \beta$ line widths (FWHM) $<4000 \mathrm{~km} \mathrm{~s}^{-1}$. In a similar spirit, Collin et al. (2006) call those galaxies emitting line profiles narrower than Gaussian profiles ( FWHM $/ \sigma_{\text {line }}<2.35$ ) Population $1 \mathrm{AGN}$ and those galaxies showing line profiles broader than Gaussian profiles Population 2 AGN (their Fig. 3). Sulentic et al. (2000) modeled the broad $\mathrm{H} \beta$ line profiles (i.e. the Population B quasars) with two Gaussian profiles, a broad one and a narrower one. In no case could single Gaussian profiles match their observed broad-line wings.

We demonstrated in Paper I that the shape (i.e. the $\mathrm{FWHM} / \sigma_{\text {line }}$ ratio) of the $\mathrm{H} \beta$, He II $\lambda 4686$, and C IV $\lambda 1550$ profiles varies systematically with their line width. There is no clear evidence for two separate quasar populations. Here we show that this applies for $\mathrm{H} \gamma, \mathrm{H} \alpha$, Ly $\alpha$, He II $\lambda 1640, \mathrm{C}$ III] $\lambda 1909$, and $\mathrm{S}$ IV $\lambda 1400$ lines in our sample as well. The observed profiles of all these emission lines can simply be characterized by rotational line broadening of Lorentzian profiles. To every emission line belongs one exclusive turbulent velocity of the line emitting region. The turbulent velocities go from $400 \mathrm{~km} \mathrm{~s}^{-1}$ for the lowionization lines up to $3800 \mathrm{~km} \mathrm{~s}^{-1}$ for the high-ionization lines (see Table 2). The rotational velocities go from $500 \mathrm{~km} \mathrm{~s}^{-1}$ up to $6500 \mathrm{~km} \mathrm{~s}^{-1}$.

Baskin \& Laor (2005) claim that narrow CIV lines are rare ( 2 per cent occurrence rate) compared with narrow $\mathrm{H} \beta<$ $2000 \mathrm{~km} \mathrm{~s}^{-1}$ ( 20 per cent) based on more than 80 spectra from the Boroson-Green sample. This can be understood easily in terms of the high turbulent velocities belonging to the C IV $\lambda 1550$ line emission region $\left(2900 \mathrm{~km} \mathrm{~s}^{-1}\right)$ in comparison to the $\mathrm{H} \beta$ line region $\left(400 \mathrm{~km} \mathrm{~s}^{-1}\right)$.

Line broadening due to turbulence and rotation are the main constituents for the observed broad-line profiles in AGN. However, inclined accretion disk geometries of the line emitting 
regions lead to smaller line widths owing to projection effects, while their $\mathrm{FWHM} / \sigma_{\text {line }}$ ratio remains constant (see Paper I). Additional asymmetries in the line profiles might be caused by geometrical/optical obscuration effects, additional outflow/inflow components, anisotropic emission, superposition of line emission from different emitting region, etc. It was mentioned in Paper I that line asymmetries lead to lower $\mathrm{FWHM} / \sigma_{\text {line }}$ ratios as well.

This general topic should be studied in more detail in the future. Furthermore, obscuration affects individual line profiles in a different way because the lines originate in different regions (see below).

\subsection{Geometry and structure of the line emitting region}

In Table 2 one can identify the clear trend for higher ionized lines to originate in those regions where higher turbulent velocities are predominant. As higher ionized lines exhibit broader emission lines in general and usually originate closer to the center (e.g. Peterson \& Wandel 1999; Kollatschny et al. 2001). This trend is consistent with a general increase in the turbulent velocity towards central regions. The broad emission lines in AGN originate at distances of less than one light day to more than 100 light days from the central ionizing source (e.g. Desroches et al. 2006; Kaspi et al. 2002; Bentz et al. 2009).

Based on the earlier theoretical studies of Pringle (1981, Eq. (3.16)), we made the following claim about the geometry of AGN accretion disks in Paper I. The ratio of the accretion disk height $\mathrm{H}$ with respect to their radius $\mathrm{R}$ is proportional to the turbulence velocity $v_{\text {turb }}$ in the accretion disk with respect to the rotational velocity $v_{\text {rot }}$

$H / R=(1 / \alpha)\left(v_{\text {turb }} / v_{\text {rot }}\right)$.

The unknown viscosity parameter $\alpha$ is assumed to be constant. We did not consider magnetic fields in this picture and made the obvious assumption that the turbulence velocity $v_{\text {turb }}$ is less than the sound of speed $c_{\mathrm{S}}$ in the disk:

$$
\begin{aligned}
& v_{\text {turb }}<c_{\mathrm{S}} \\
& v_{\text {turb }}=\alpha^{\prime} c_{\mathrm{S}}
\end{aligned}
$$

where $\alpha^{\prime}$ is close to the usual $\alpha$ parameter.

The rotational velocities - belonging to the individual emission lines - vary by a factor of more than ten, while the turbulent velocities that are connected with the individual emission lines remain constant. This can be seen in Fig. 7, as well as in Figs. 11 to 16. In this accretion disk model and accepting our results breaking the velocity degeneracies one would conclude that slow-rotating AGN host a thick accretion disk that is ten times thicker than fast-rotating AGN. A schematic picture of thick and thin accretion disks is shown in Fig. 17. The radius of the central black hole $\left(r=5.9 \times 10^{12} \mathrm{~cm}\right)$ in Fig. 17 corresponds to a Schwarzschild mass of $M=2 \times 10^{7} M_{\odot}$.

Other details regarding the physical conditions in the line emitting region seem to be even more complex: it has been noticed by means of reverberation measurements that the He II $\lambda 4686$ and He II $\lambda 1640$ lines in AGN spectra originate at different distances from the center (e.g. Peterson et al. 2004), although they hold the same ionization degree. Diverse models do not reproduce this observational fact (e.g. Bottorff 2002). Now we independently confirm the former finding that these lines originate in different physical regions based on the distinct turbulent velocities we deduced for the two helium II line emission regions. This is important considering that the
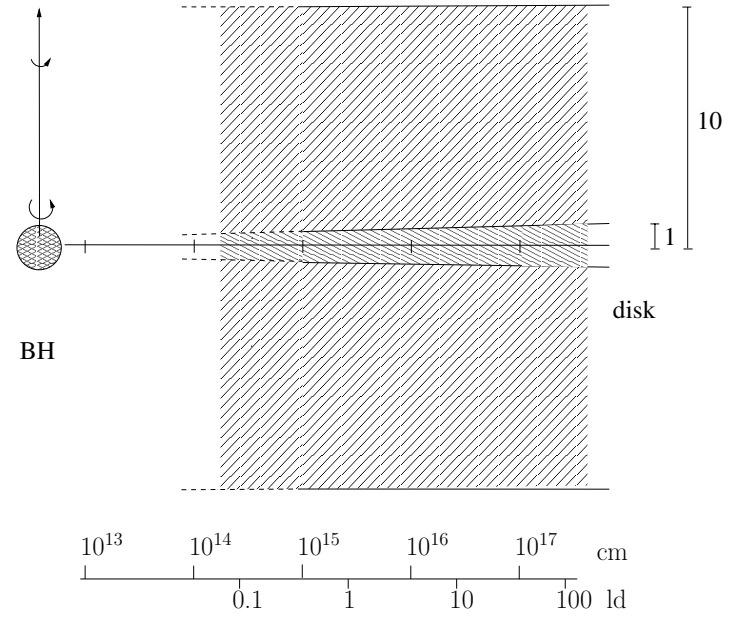

Fig. 17. Schematic accretion disk models of AGN showing that slowrotating AGN have an accretion disk that is ten times thicker than fastrotating $\mathrm{AGN}$.

He II $\lambda 4686 / \mathrm{He}$ II $\lambda 1640$ ratio has sometimes been used as a reddening indicator for the broad-line region (e.g. Snijders et al. 1986; Ferguson et al. 1995).

The eigenvector studies of Boroson \& Green (1992) demonstrated a strong correlation between the emission line width, optical FeII emission, and soft X-ray photon index in AGN (Eigenvector 1). The line width is connected with the geometry or rather, to be more specific, with the thickness of the accretion disk (see above). Therefore, the observed strength of the spectral FeII emission and the X-ray photon index might be affected by geometry effects as well.

\subsection{Correction factors for calculating central black hole masses}

The central black hole mass $M_{\mathrm{BH}}$ in AGN can be derived from the broad emission line widths under the assumption that the gas dynamics are dominated by the central massive object:

$M_{\mathrm{BH}}=f c \tau_{\text {cent }} \Delta v^{2} G^{-1}$.

The characteristic distances of the line-emitting regions $c \tau_{\text {cent }}$ from the central ionizing source can be derived by means of spectroscopic variability campaigns. The distance can be calculated from the cross-correlation function of emission line intensity variations with respect to the ionizing continuum intensity variations (e.g. Koratkar \& Gaskell 1991; Kollatschny \& Dietrich 1997). The characteristic velocity $\Delta v$ of the emission line region can be estimated from the FWHM of the rms profile or from the line dispersion $\sigma_{\text {line }}$ (e.g. Peterson et al. 2004). The scale factor $\mathrm{f}$ depends among others on the geometry and structure of the line emitting region, as well as on their inclination. Scale factors for rms spectra have numerical values from 0.5 up to 6.2 (e.g. Collin et al. 2006), depending on the assumptions about geometry and structure of the line emitting region. Only very few cases are known where the black hole mass could be estimated independently by, e.g., gravitational redshift measurements (Kollatschny 2003b) to get additional information on the scale factor $f$ or rather the inclination angle.

Variability campaigns are very expensive with respect to their observing time. Kaspi et al. (2005) verify a relationship between AGN luminosity and Balmer-line-averaged BLR size based on variability campaigns. This scaling relation can be used 


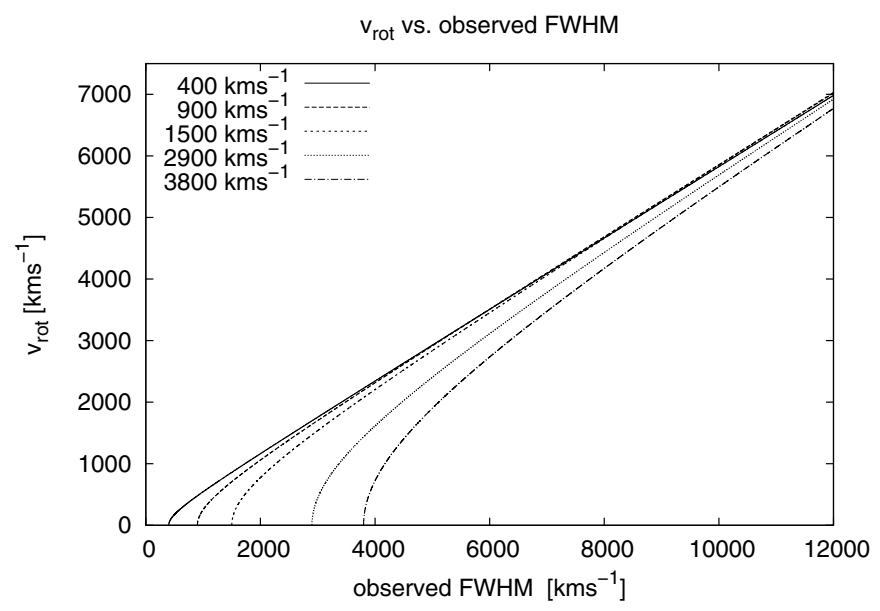

Fig. 18. Relation between observed FWHM of the emission line profiles and the related rotational velocity $v_{\text {rot }}$. The relation is shown for intrinsic turbulence profiles with $v_{\text {turb }}$ ranging from $400 \mathrm{~km} \mathrm{~s}^{-1}$ up to $3800 \mathrm{~km} \mathrm{~s}^{-1}$.

to obtain black hole masses based on single epoch spectra alone. The velocity of the line emitting region can be estimated on the basis of the width of the Balmer emission lines. This method was extended to other emission lines, especially to the C IV $\lambda 1549$ line of distant high-redshift AGN.

Central black hole masses were generally found to be bigger for AGN showing broad $\mathrm{H} \beta$ profiles (Pop. B), as well as broad CIV $\lambda 1550$ profiles than for those AGN that exhibit narrow $\mathrm{H} \beta$ profiles (Pop. A) (Marziani et al. 2003; Peterson et al. 2004; Vestergaard 2004; Zamfir et al. 2010).

The broader emission lines usually originate closer towards the center as has been noted before (e.g. Peterson et al. 2004; Kollatschny 2003a). The turbulence grows towards the inner zones as well. Therefore the black hole mass estimates, based on the line widths, are more heavily biased in broad emission line objects caused by the additional turbulence broadening than in the narrower emission line objects.

We consider here nearby AGN where we have good black hole mass estimations from reverberation mapping. Shen et al. (2008) have investigated biases in virial black hole masses based on SDSS spectra. Wang et al. (2011) discuss the influence of radiation-driven outflows on CIV emission line profiles in highredshift and high-luminosity AGNs. In the case of such outflows, the CIV emission lines should become broader because of the additional line broadening component of the outflow. If these CIV profiles of high-redshift and high-luminosity AGNs show the same widths as those of nearby AGNs, then the contribution of the rotation on the line width must be even lower. In that case the black hole mass estimation that is only based on the rotation velocity must be scaled down even more.

Vestergaard (2004) derived black hole masses of nearby quasars based on their $\mathrm{H} \beta$ line widths, as well as black hole masses of high-redshift quasars based on their C IV $\lambda 1549$ line widths.

On average, she found bigger black hole masses - by a factor five to ten - for the younger high-redshift quasars based on their C IV $\lambda 1549$ line widths in comparison to the old nearby quasars based on their $\mathrm{H} \beta$ line widths. In an additional paper, Vestergaard \& Peterson (2006) published different scaling relations for nearby and distant AGN black hole masses based on their $\mathrm{H} \beta$, as well as $\mathrm{C}$ IV $\lambda 1549$ line widths. However, Netzer et al. (2007) note that using the C IV $\lambda 1549$ line width for estimating black hole masses gives considerably different results

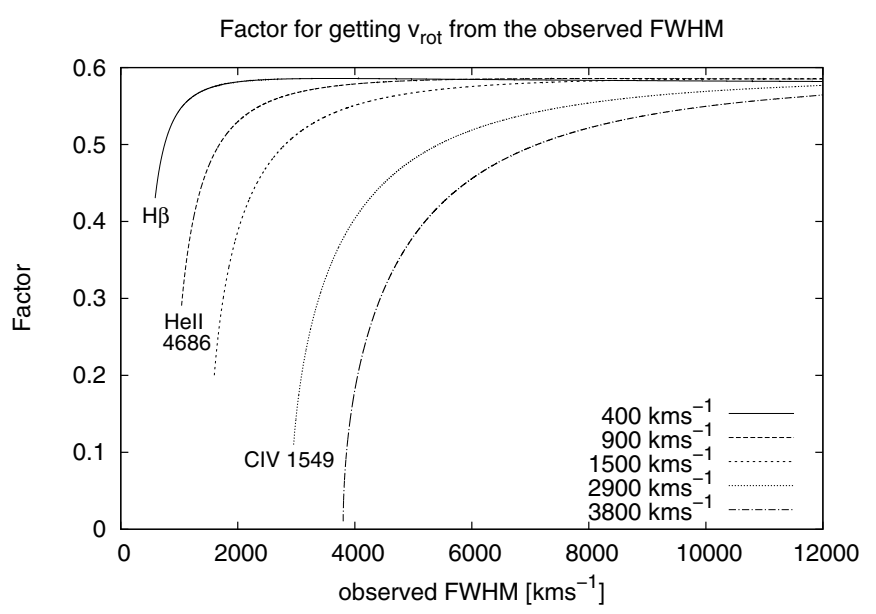

Fig. 19. Factor for getting the rotational velocity $v_{\text {rot }}$ of the line emitting region from the observed FWHM, shown for observed profiles having intrinsic turbulence velocities ranging from $400 \mathrm{~km} \mathrm{~s}^{-1}$ up to $3800 \mathrm{~km} \mathrm{~s}^{-1}$.

and a larger scatter than using the $\mathrm{H} \beta$ line. We demonstrated in Paper I that black hole mass estimates based on the line width of the C IV $\lambda 1549$ line are overestimated by a factor of five to ten in comparison to those black hole masses based on the width of the $\mathrm{H} \beta$ line.

On the basis of our observed and modeled line width ratios in Figs. 7, 11-16, we present in Fig. 18 the relation between the observed FWHM of the emission line profiles and the related rotational velocity $v_{\text {rot }}$. This relation is shown for underlying turbulence profiles with $v_{\text {turb }}$ ranging from $400 \mathrm{~km} \mathrm{~s}^{-1}$ to $3800 \mathrm{~km} \mathrm{~s}^{-1}$. In Fig. 19 we present the correction factor towards the rotational velocity $v_{\text {rot }}$ of the broad-line clouds to calculate the central black hole masses of AGNs based on the observed line widths.

Computed black hole masses go with the square of the rotational velocity (see Eq. (6)). Therefore, the corrected intrinsic black hole masses should be lower by a factor of two to more than ten with respect to the calculated black hole masses that have not been corrected for the turbulent velocity contribution. This correction factor is different for the different emission lines (depending on the underlying turbulent velocity of the individual lines) and also depends on the additional rotational velocity broadening. There is not an exclusive correction factor for black hole mass estimates based on the $\mathrm{C}$ IV $\lambda 1549$ or $\mathrm{H} \beta$ line widths as proposed by Vestergaard \& Peterson (2006) in their mass scaling relations. Furthermore, black hole masses of distant AGN based on the C IV $\lambda 1549$ line widths have been overestimated in comparison to nearby AGN where the mass estimate is based on the $\mathrm{H} \beta$ line. It is important to consider this effect for our general understanding of the evolutionary history of black hole masses in AGN.

\section{Conclusion}

We investigated the profile shapes of the UV/optical broad emission lines in AGN in detail. The two basic components causing the line profile are Lorentzian profiles and rotational broadening. An intrinsic turbulent velocity belongs to each specific AGN emission line, which causes different line widths (FWHM) of the particular Lorentzian profiles. The turbulent velocities go from $400 \mathrm{~km} \mathrm{~s}^{-1}$ for $\mathrm{H} \beta$ up to $3800 \mathrm{~km} \mathrm{~s}^{-1}$ for $\mathrm{Ly} \alpha+\mathrm{N} \mathrm{v} \lambda 1240$. The rotation velocities causing the line profile broadening go from $500 \mathrm{~km} \mathrm{~s}^{-1}$ up to $6500 \mathrm{~km} \mathrm{~s}^{-1}$. There are not two separated classes of broad-line AGN (narrow line and broad-line objects), but instead a continuous transition from narrow to broad-line 
objects. The accretion disk thickness in AGN can be derived from the ratio of the turbulent velocity $v_{\text {turb }}$ with respect to the rotational velocity $v_{\text {rot }}$. Slow-rotating AGN have an accretion disk that is ten times thicker than fast-rotating AGN. We find clear evidence that the He II $\lambda 4686$ and He II $\lambda 1640$ lines originate in different physical regions in AGNs, although they hold the same ionization degree. This finding is based on the distinct turbulent velocities we deduce for the two helium II line emission regions.

Black hole mass corrections resulting from turbulent velocity considerations are nontrivial and are larger for C IV $\lambda 1549$ based measurements than $\mathrm{H} \beta$, and both are a function of the observed FWHM. In the literature usually the widths of the broad emission lines are used to compute the central black hole masses in AGN. However, one has to consider the contribution by the turbulence to get the corrected intrinsic rotational velocities. This investigation presented the individual correction factors towards the different emission lines for getting the intrinsic rotational velocities $v_{\text {rot }}$ of the line emitting region from the observed FWHMs. The corrected black hole masses are lower than the uncorrected black hole masses by a factor of two to more than ten, depending on the emission lines, as well as on the rotational velocities. Especially those masses of the distant AGN have been grossly overestimated where the masses have been estimated on the basis of the C IV $\lambda 1549$ line widths.

Acknowledgements. This work has been supported by the Niedersachsen-Israel Research Cooperation Program ZN2318 and DFG grant Ko 857/32-1.

\section{References}

Baskin, A., \& Laor, A. 2005, MNRAS, 356, 1029

Bentz, M. C., Walsh, J. L., Barth, A. J., et al. 2009, ApJ, 705, 199

Blumenthal, G. R., \& Mathews, W. G. 1975, ApJ, 198, 517

Boroson, T., \& Green, R. 1992, ApJS, 80, 109

Bottorff, M., C., Baldwin, J. A., Ferland, G. J., et al. 2002, ApJ, 581, 932

Capriotti, E., Foltz, C., \& Byard, P. 1980, ApJ, 241, 903

Collin, S., Kawaguchi, T., Peterson, B. M., \& Vestergaard, M. 2006, A\&A, 456, 75

Collin-Souffrin, S., Dyson, J. E., McDowell, J. C., \& Perry, J. J. 1988, MNRAS, 232,539

Desroches, L.-B., Filippenko, A. V., Kaspi, S., et al. 2006, ApJ, 650, 88
Elvis, M. 2000, ApJ, 545, 63

Emmering, R. T., Blandford, R. D., \& Shlosman, I. 1992, ApJ, 385, 460 Ferguson, J. W., Ferland, G. J., \& Pradhan, A. K. 1995, ApJ, 438, L55 Fromerth, M. J., \& Melia, F. 2000, ApJ, 533, 172

Gaskell, C. M. 2010, ASP Conf. Ser., 427, 68

Goad, M. R., Korista, K. T., \& Ruff, A. J. 2012, MNRAS, 426, 3086

Ho, L. 2008, ARA\&A, 45, 475

Hu, C., Wang, J.-M., Ho, L. C., et al. 2012, ApJ, 760, 126

Hubeny, I., Lanz, T., \& Jeffrey, C. S. 1994, in Newsletter of Astronomical Spectra No. 20., ed. C. S. Jeffrey (St. Andrews Univ.)

Kaspi, S., Smith, P. S., Netzer, H., et al. 2002, ApJ, 533, 631

Kaspi, S., Maoz, D., Netzer, H., et al. 2005, ApJ, 629, 61

Königl, A., \& Kartje, J. E. 1994, ApJ, 434, 446

Kollatschny, W. 2003a, A\&A, 407, 461

Kollatschny, W. 2003b, A\&A, 412, L61

Kollatschny, W., \& Dietrich, M. 1997, A\&A, 323, 5

Kollatschny, W., \& Zetzl, M. 2011, Nature, 470, 366 (Paper I)

Kollatschny, W., Bischoff, K., Robinson, E. L., et al. 2001, A\&A, 379, 125

Koratkar, A., \& Gaskell, M. 1991, ApJ, 370, L61

Laor, A. 2006, ApJ, 643, 112

Marziani, P., Zamanov, R. K., Sulentic, J. W., et al. 2003, MNRAS, 345, 1133

Moriya, T. J., \& Tominaga, N. 2012, ApJ, 747, 118

Murray, N., \& Chiang, J. 1997, ApJ, 474, 91

Netzer, H. 1990, in 20 Saas-Fee Advanced Course of the Swiss Society for Astrophysics and Astronomy: Active Galactic Nuclei (Berlin: Springer), 57

Netzer, H., Lira, P., Trakhtenbrot, B., et al. 2007, ApJ, 671, 1256

Osterbrock, D. E. 1978, Proc. National Acad. Sci., 75, 540

Peterson, B. M., \& Wandel, A. 1999, ApJ, 521, L95

Peterson, B. M., \& Wandel, A. 2000, ApJ, 540, L13

Peterson, B. M. Ferrarese, L., Gilbert, K. M., et al. 2004, ApJ, 613, 682

Pringle, J. E. 1981, ARA\&A, 19, 137

Richards, G. T., Vanden Berk, D. E., Reichard, T. A., et al. 2002, AJ, 124, 1

Richards, G. T., Kruczek, N. E., Gallagher, S. C., et al. 2011, AJ, 141, 167

Robinson, A. 1995, MNRAS, 272, 647

Shen, Y., Greene, J. E., Strauss, M. A., et al. 2008, ApJ, 680, 169

Snijders, M. H. J., Netzer, H., \& Boksenberg, A. 1986, MNRAS, 222, 549

Sulentic, J. W., Marziani, P., \& Dultzin-Hacyan, D. 2000, ARA\&A, 38, 521

Sulentic, J. W., Marziani, P., Zamanov, R., et al. 2002, ApJ, 566, L71

Sulentic, J. W., Marziani, P., Zamfir, S., \& Meadows, Z. A. 2012, ApJ, 752, L7

Unsoeld, A. 1955, in Physics of stellar atmospheres (Springer)

Urry, C. M., \& Padovani, P. 1995, PASP, 107, 803

Veron-Cetty, M.-P., Veron, P., Goncalves, A. C. 2001, A\&A, 372, 730

Vestergaard, M. 2004, ApJ, 601, 676

Vestergaard, M., \& Peterson, B. M. 2006, ApJ, 641, 689

Wang, H., Wang, T., Zhou, H., et al. 2011, ApJ, 738, 85

Zamfir, S., Sulentic, J. W., Marziani, P., \& Dultzin, D. 2010, MNRAS, 403, 1759 